\title{
A randomised trial of effects of alcohol on cytochrome P450 eicosanoids, mediators of inflammation resolution and blood pressure in men.
}

Anne E. Barden $\mathrm{PhD}^{1}$, Venus Chavez BSc (Hons) ${ }^{1}$, Michael Phillips MSc ${ }^{2}$, Emilie Mas $\mathrm{PhD}^{1}$, Lawrence J Beilin MD FRACP ${ }^{1}$, Kevin D Croft PhD ${ }^{1}$, *Trevor A Mori PhD ${ }^{1}$, *Ian B Puddey MD FRACP ${ }^{1}$

*Joint senior authors

University of Western Australia, School of Medicine and Pharmacology, Royal Perth Hospital ${ }^{1}$, University of Western Australia, Harry Perkins Research Institute of Medical Research $^{2}$, Perth Australia

\section{Sources of support}

This study was funded from project grants from the National Health and Medical Research Council of Australia and the Royal Perth Hospital Medical Research Foundation.

Orlando Wines, South Australia, supplied the red wine and de-alcoholised red wine used in this study. We thank Noelene Atkins and Adeline Indrawan for their assistance with volunteer recruitment and analysis of cytochrome P450 metabolites, respectively.

Correspondence to Professor Anne Barden,

School of Medicine \& Pharmacology,

Level 4 MRF Building, Rear 50 Murray St, Perth, WA 6000, Australia.

Tel: +61 89224 0272; Fax: +61 89224 0246; e-mail: anne.barden@uwa.edu.au 


\begin{abstract}
Background: Cardiovascular effects of alcohol consumption may be influenced by both pro- and anti-inflammatory mechanisms. We previously showed that chronic alcohol consumption increased blood pressure (BP), oxidative stress and 20hydroxyeicosatetraenoic acid (20-HETE), a vasoconstrictor and pro-inflammatory eicosanoid synthesised by CYP450 enzymes from arachidonic acid. This study in men examined the effect of consuming red wine (RW) on BP in relation to changes in 20HETE, oxidative stress ( $\mathrm{F}_{2}$-isoprostanes), markers of inflammation, anti-inflammatory CYP450 epoxyeicosatrienoic acids (EETs) and specialised pro-resolving mediators of inflammation (SPMs) derived from eicosapentaenoic acid (EPA) and docosahexaenoic acid (DHA).
\end{abstract}

Methods: Normotensive men $(n=22)$ were randomly allocated to drink RW (375ml/day) or the equivalent volume of de-alcoholised red wine (DRW) or water for 4 weeks in a 12 week, 3-period crossover trial. BP, heart rate, 20-HETE, F2-isoprostanes and SPM were measured at baseline, 4,8 , and 12 weeks.

Results: Drinking RW increased BP $(P<0.05)$, plasma and urinary 20-HETE $(P<0.05)$, plasma $\mathrm{F}_{2}$-isoprostanes $(P<0.0001)$, and the SPMs 18 -hydroxyeicosapentaenoic acid (18-HEPE) from EPA, and resolvin D1 (RvD1) and 17R-resolvin D1 (17R-RvD1) from DHA (all $P<0.05$ ) compared with DRW and water. EETs and hs-CRP were unaffected by RW. Plasma 18-HEPE was positively related to urinary 20-HETE $(\mathrm{P}<0.008)$ only after RW.

Conclusion: This study has shown that men consuming moderate-to-high alcohol as red wine for 4 weeks, had increased BP, 20-HETE and oxidative stress, as well as specific SPM that resolve inflammation. These paradoxical findings require further studies to 
determine whether alcohol stimulates different CYP450 enzymes and whether the findings can be replicated in females.

KEY WORDS: alcohol, blood pressure, 20-hydroxyeicosatetraenoic acid, oxidative stress, resolvins 


\section{INTRODUCTION}

Moderate-to-high alcohol consumption is a major contributor to elevated blood pressure (BP) (Klatsky, 2015). However, the mechanisms underlying this association are not fully understood. In a cross-sectional study of 69 treated hypertensives we showed a significant positive correlation between usual alcohol intake and urinary 20hydroxyeicosatetraenoic acid (20-HETE) excretion (Ward et al., 2005). 20-HETE is a potent vasoconstrictor eicosanoid derived from cytochrome P450 $\omega$-hydroxylase metabolism of arachidonic acid (AA). AA is also metabolised by CYP450 epoxygenases producing 4 epoxyeicosatrienoic acids (EETs) (5,6-EET; 8,9-EET; 11,12EET \& 14,15-EET) (Roman, 2002). The EETs, that are vasodilatory and antiinflammatory, are metabolised to their inactive dihydroxyeicosatrienoic acids (DHETs) by soluble epoxide hydrolase (Figure 1). We subsequently showed that reducing beer intake by $\sim 60 \mathrm{~g}$ alcohol/day for 4 weeks in 16 healthy males substantially reduced $24 \mathrm{~h}$ urinary 20-HETE, raising the possibility that altered 20-HETE synthesis may constitute an important mechanism for the development of alcohol-related hypertension (Barden et al., 2007). In the same study, reducing beer intake significantly reduced plasma total $\mathrm{F}_{2}-$ isoprostanes (Barden et al., 2007) that are markers of in vivo oxidative stress formed via non-enzymatic free radical attack on AA (Figure 1).

Imhof et al. (2001) showed a U-shaped relationship between alcohol consumption and markers of inflammation. In a population study, non-drinkers and heavy drinkers had higher $\mathrm{C}$ reactive protein (CRP) concentrations than moderate drinkers (Imhof et al., 2001). The effect of alcohol to increase 20-HETE may affect vascular inflammation. 20HETE can activate NF- $\kappa \mathrm{B}$ and the MAPK/ERK signalling pathways, inducing proinflammatory cytokines and transforming endothelium to an inflammatory phenotype 
(Hoopes et al., 2015; Ishizuka et al., 2008). 20-HETE has been implicated in the vascular inflammation associated with ischaemia/reperfusion injury (Hoff et al., 2011) and cerebrovascular inflammation in the spontaneously hypertensive rat (Toth et al., 2013).

Alcohol also has the potential to alter inflammation resolution, a process that is driven by specialised pro-resolving mediators of inflammation (SPMs). The SPM's include lipoxins from AA and resolvins, protectins and maresins derived from eicosapentaenoic acid (EPA) and docosahexaenoic acid (DHA). SPMs have been shown to halt neutrophil infiltration, and enhance macrophage uptake of apoptotic cells and debris, promoting a return to homeostasis. (Barden et al., 2016; Serhan et al., 2015a). Alcohol could affect synthesis of SPMs by affecting the availability of fatty acid substrates (Kim et al., 2007), and altering the activity of key enzymes involved in SPM synthesis, namely cytochrome P450 (Salmela et al., 1998), COX-2 (Knapp and Crews, 1999) and 5lipoxygenase (Wheelan and Murphy, 1997) (Figure 1).

This study aimed to examine the effect of consuming red wine on BP in moderate- to heavy-drinking, but otherwise healthy men, in relation to changes in 20-HETE and EETs, oxidative stress, markers of inflammation and SPM. We hypothesised that chronic alcohol consumption would increase BP, 20-HETE, markers of inflammation and oxidative stress, and attenuate SPM.

\section{METHODS}

\section{Recruitment and study design.}

\section{Study Participants}


Healthy males aged 20-65 years, who were regular drinkers consuming between 40 $100 \mathrm{~g}$ alcohol/day, were sought by advertisement from the general population in Perth, Western Australia between 2008 and 2009. Women were excluded from the study because the protocol required that a relatively high daily dose of alcohol be consumed. Participants had a body mass index $(\mathrm{BMI})<30 \mathrm{~kg} / \mathrm{m}^{2}$, and no history of liver disease, cardiovascular disease, diabetes mellitus, hypertension or dyslipidaemia. They were non-smokers, not taking medication including aspirin or nonsteroidal anti-inflammatory drugs. A questionnaire assessed previous alcohol consumption detailing the type of beverage (beer; wine; and spirits) frequency of consumption (every day; 5-7 times/wk; 1-4 times/wk; $1-4$ times/month; $<1$ time/month) and the duration of consumption $(<1$ year; 1-2 years; 2-5 years and >5 years). At a screening visit, six BP readings were obtained at 1-min intervals, after 5-min seated rest, using a Dinamap Pro 200 (GE Medical Systems, Tampa, Florida. USA) and an ECG recording was obtained. Blood was collected for measurement of glucose, lipids and liver function. A medical examination by a physician determined each volunteer's suitability for the study. The physical examination excluded volunteers that had any pathology (neural, hepatic or cardiac) that might characterise them as alcoholics but no formal questionnaire was administered to assess alcoholism. The level of alcohol exposure during the study was at the upper limit for men defined by National Health and Medical Research Council of Australia guidelines at the time of the study.

\section{Study Design}

During a 2-week run-in period, participants were asked to abstain from drinking alcohol. Baseline measurements were then obtained, and they entered a 3-period open 
label crossover trial. Each period was 4 weeks in duration with no washout between interventions. During the 3 study periods they consumed either red wine (RW) (375ml/day, 41g alcohol) or the equivalent volume of de-alcoholised red wine (DRW) or water (W) in an unblended study. They were instructed to drink the beverages provided with their evening meal and to refrain from drinking any other alcohol for the 12 weeks of the study. They were allocated to each study period sequence via block randomization using computer-generated random numbers, devised by the statistician. The RW and DRW were supplied by Orlando Wines, South Australia, and had an alcohol and polyphenol content of $\sim 14 \%$ and $2378 \mathrm{mg} / \mathrm{L}$ and $0.6 \%$ and $2284 \mathrm{mg} / \mathrm{L}$, respectively. Volunteers were asked to maintain their usual diet and exercise throughout the study with emphasis not to alter consumption of tea and coffee, a rich source of polyphenols. Researchers entering BP and biochemical data or performing laboratory analyses were blinded to the treatment sequences.

The study compared effects of RW with DRW and water on CYP450 eicosanoids and blood pressure (primary outcomes) and $\mathrm{F}_{2}$-Isoprostanes and SPM (secondary outcomes). The trial was conducted according to the Declaration of Helsinki guidelines and approved by the University of Western Australia Human Research Ethics Committee. Written informed consent was obtained from all participants. The trial was registered with the Australian New Zealand Clinical Trials Registry (ANZCTR), ACTRN12608000207314.

\section{BP Monitoring}

Ambulatory BP was measured after the two-week run-in period (baseline) and after each of the 4-week treatment periods. An ambulatory BP monitor (Spacelabs 90207, Spacelabs Healthcare, Issaquah, WA, USA) was fitted to the non-dominant arm by a 
trained researcher who explained its use to the participants. BP and heart rate were monitored over $24 \mathrm{~h}$ at 20 -minute intervals while awake and at 30 -minute intervals while asleep. Volunteers were instructed to continue their usual daily activities, avoid any vigorous exercise, and to keep a diary documenting when they were awake or asleep. Measurements showing an error code or those with a pulse pressure $<20 \mathrm{~mm} \mathrm{Hg}$ were excluded from the analysis. BP measurements were considered complete if $>80 \%$ of the recordings were valid. BP readings from each subject were aggregated to calculate average hourly results.

\section{Measurement of Compliance and Lifestyle Change}

A 7-day retrospective alcohol diary was completed each week to monitor alcohol intake. A fasting blood sample was provided at the end of the 2-week run-in period and the end of each study period for $\gamma$-glutamyl transpeptidase as a biomarker of change in alcohol intake, and total cholesterol, triglycerides, high-density lipoprotein cholesterol (HDL-C) and low-density lipoprotein cholesterol (LDL-C). Volunteers were advised not to change their usual dietary habits or level of physical activity throughout the study. Questionnaires were administered throughout the study to evaluate changes in diet including major food groups, tea, coffee and alcohol consumption, as well as any changes in the type and amount of physical activity. Body weight was monitored throughout the study. Urinary 4-O-methylgallic acid was measured as a biomarker of red wine polyphenol intake (AbuAmsha et al., 1996) .

\section{Biochemical analyses}

A fasted blood sample and 24-hr urine collection were collected at baseline, 4, 8 and 12 weeks. Serum samples were analysed by PathWest Laboratories, Royal Perth Hospital, using the Hitachi 917 Biochemical Analyser (Hitachi Limited, Tokyo, Japan). $\gamma$ - 
glutamyl transpeptidase was measured with a Roche kit (Roche Diagnostics GmbH, D68298, Mannheim, Germany). HDL-C was measured with the Boehringer Mannheim kit. LDL-C was calculated using the Friedewald formula (Friedewald.WT et al., 1972). Hs-CRP was measured by nephelometry at PathWest Laboratories. Aliquots from 24hour urine collections stored at $-80^{\circ} \mathrm{C}$ were assayed for $4-O$-methylgallic acid in our laboratory by gas chromatography-mass spectrometry (GCMS) (Caccetta et al., 2000). All samples from each subject were assayed in the one batch.

\section{Measurement CYP450 eicosanoids and F2-Isoprostanes}

Plasma and urinary 20-HETE (Barden et al., 2013), plasma total EETs and DHETs (Barden et al., 2013), and plasma and urinary $\mathrm{F}_{2}$-isoprostanes (Tsai et al., 2009) were measured in our laboratory by GCMS as previously described, with the technician blinded to the allocation sequence of the study participants.

\section{Measurement of Plasma SPMs}

Venous blood (10ml) from the antecubital fossa, was collected on ice into EDTA (2.5mM), butylated hydroxytoluene $(0.18 \mathrm{mM})$ and reduced glutathione $(3.3 \mathrm{mM})$, centrifuged at $4^{\circ} \mathrm{C}$, and the plasma was stored at $-80^{\circ} \mathrm{C}$ until analysis. SPMs were measured in plasma $(1 \mathrm{ml})$ using liquid chromatography tandem mass spectrometry (LCMSMS) as previously described (Barden et al., 2014; Mas et al., 2012). The retention time of standards, their mass spectrum and parent and product ions and collision energy were used to identify individual SPM. Standards of SPMs derived from EPA were 18R/S-hydroxy-eicosapentaenoic acid (18R/S-HEPE), resolvin E1 (RvE1), resolvin E2 (RvE2), resolvin E3 (RvE3) and 18R-resolvin E3 (18R-RvE3). Standards of SPMs derived from DHA were 17S-hydroxy-docosahexaenoic acid (17S-HDHA), resolvin D1 (RvD1), 17R-resolvin D1 (17R-RvD1), resolvin D2 (RvD2), 10S, 17S- 
dihydroxy-docosahexaenoic acid (10S,17S-DHDHA), ( \pm )14-hydroxy-docosahexaenoic acid (14R/S-HDHA) and maresin-1 (MaR-1) and protectin-1 (PD1).

\section{Statistical Analysis}

An initial power analysis on the primary outcomes, urinary 20-HETE and BP, determined $20-25$ participants would give $80 \%$ power to detect a $20 \%$ difference in urinary 20-HETE and a $5 \mathrm{mmHg}$ difference in SBP at a significance level of $\mathrm{P}<0.05$. The study was powered to detect the secondary outcome differences of $20 \%$ for plasma $\mathrm{F}_{2}$-isoprostanes and 18-HEPE based upon repeated measures ANOVA analysis.

In the final data analysis, changes in SBP, DBP and heart rate, plasma and urine CYP450 eicosanoids and $\mathrm{F}_{2}$-isoprostanes, and plasma SPMs were examined using linear mixed model analysis adjusted for baseline measurements and compared for each beverage using linear mixed models with restricted maximum likelihood estimation. The changes to the initial analysis plan were made because of the gain in statistical power of mixed/multilevel analysis. A covariate was entered into each of the regression analyses to test for the effects of the randomization order on each of the outcome variables. The relationship between urinary 20-HETE and plasma 18-HEPE was examined using a linear mixed model that adjusted for baseline measurements and used an interaction term to test for differences in the slope of relationship attributed to each beverage.

\section{RESULTS}

Seventy-one men were phone screened, and 30 attended the research unit for anthropometry, BP measurement, biochemical screening and a physical examination. Twenty-two men who were moderate-to-high alcohol drinkers commenced and 
completed the study. Eleven of the men reported the duration of their current alcohol consumption was $<5$ years and eleven $\geq 5$ years. They were middle aged and slightly overweight, but otherwise healthy, with lipids and glucose within the normal range for adults (Table 1). Eighty-six percent were pre-hypertensive (SBP between $120 \mathrm{mmHg}$ and $139 \mathrm{mmHg}$ ). Compliance with alcohol consumption during the study assessed by alcohol diaries and $\gamma \mathrm{GT}$ was excellent. Alcohol intake assessed from alcohol diaries was 45.4g/day after drinking RW, compared with 7.9g/day after DRW and 4.9g/day after water. $\gamma \mathrm{GT}$ a marker of alcohol intake was significantly elevated after drinking RW $(33.1 \pm 4.1 \mathrm{U} / \mathrm{L}, P<0.05)$ compared with DRW $(28.7 \pm 4.1 \mathrm{U} / \mathrm{L})$ and water $(29.0 \pm 4.0$ U/L). Urinary 4-O-methylgallic acid, as a measure of compliance with consumption of RW and DRW, was similar during the RW $(1821 \pm 455 \mu \mathrm{g} / 24 \mathrm{hr})$ and DRW (1680 \pm $346 \mu \mathrm{g} / 24 \mathrm{hr}$ ) treatment periods and significantly elevated compared with the period of water consumption $(672 \pm 234 \mu \mathrm{g} / 24 \mathrm{hr}, P<0.01)$.

Total cholesterol, LDL-C and triglycerides were not differentially affected by consumption of any of the beverages (Data not shown). In contrast HDL-C was significantly elevated after RW $(1.4 \pm 0.1 \mathrm{mmol} / \mathrm{L}, P<0.05)$ compared with DRW $(1.2 \pm$ $0.1 \mathrm{mmol} / \mathrm{L})$ and water $(1.2 \pm 0.1 \mathrm{mmol} / \mathrm{L})$. Body weight did not change throughout the study and there were no changes in diet or exercise and no reported side effects of any of the treatments.

\section{Blood Pressure and Heart Rate}

After adjusting for baseline BP, drinking RW for 4 weeks increased 24-hr ambulatory BP compared with DRW and water $(\mathrm{SBP}+1.4 \pm 0.5 \mathrm{mmHg}, P=0.003$ and $\mathrm{DBP}+0.8 \pm$ $0.4 \mathrm{mmHg}, P=0.032$ ) (Table 2, Figure 2). This was mainly due to an increase in BP 
during awake hours $(\mathrm{SBP}+1.6 \pm 0.6 \mathrm{mmHg}, P=0.003$ and $\mathrm{DBP}+1.0 \pm 0.4 \mathrm{mmHg}$, $P=0.02$ ) (Table 2, Figure 2). Heart rate was increased over the $24 \mathrm{hr}$ after RW compared with DRW $(+4.8 \pm 0.4$ beats/min, $P<0.0001)$ after adjusting for heart rate at baseline. Heart rate was increased by $6.9 \pm 0.6$ beats $/ \mathrm{min}, P=0.001$ when asleep and by $3.8 \pm 0.3$ beats/min, $P=0.001$ while awake (Table 2, Figure 2). After 4 weeks drinking DRW, 24$\mathrm{hr}$ heart rate was reduced compared with the period of drinking water $(-2.0 \pm 0.4$ beats $/ \min , P=0.001)$, and was lower while awake $(-2.1 \pm 0.6$ beats $/ \min , P=0.001)$ and while asleep $(-1.8 \pm 0.7$ beats/min, $P=0.006)$ (Table 2, Figure 2).

\section{CYP450 eicosanoids}

Urinary 20-HETE was higher after consuming RW when compared with DRW $(P<0.0001)$ and water $(P=0.0003)$ (Figure 3a, Supplementary Table 1). Plasma 20HETE was higher after RW compared with DRW ( $P=0.049$ ) (Figure 3b, Supplementary Table 1), but EETs and DHETS were not significantly different across the 3 treatment periods (Figure 3c and 3d, Supplementary Table 1). The effect of 4 weeks consumption of DRW on urinary 20-HETE and plasma 20-HETE, EETs and DHETs was not different from drinking water (Figure 3a-d).

\section{Plasma and urinary $\mathbf{F}_{2}$-Isoprostanes}

Plasma $\mathrm{F}_{2}$-isoprostanes were elevated after consumption of RW compared with DRW $(P<0.0001)$ and water $(P<0.0001)$ (Figure $3 e$, Supplementary Table 1$)$. Urinary $\mathrm{F}_{2}-$ isoprostanes were not different across the 3 treatment periods (Figure 3f, Supplementary Table 1). The effect of 4 weeks consumption of DRW on plasma and urinary $F_{2-}$ isoprostanes was not different from drinking water.

\section{Markers of inflammation and resolution of inflammation}

Hs-CRP was not altered by any of the beverages and was $1.36 \pm 0.30 \mathrm{mg} / \mathrm{L}$ at baseline, 
$1.14 \pm 0.30 \mathrm{mg} / \mathrm{L}$ after water; $1.12 \pm 0.30 \mathrm{mg} / \mathrm{L}$ after $\mathrm{DRW}$ and $1.27 \pm 0.30 \mathrm{mg} / \mathrm{L}$ after red wine. In contrast, several SPMs were significantly affected by consumption of RW. Plasma 18-HEPE derived from EPA, and RvD1 and 17R-RvD1 from DHA, were significantly elevated $(P<0.05)$ after consumption of RW compared with DRW and water (Figure 4, Supplementary Table 1). 17-HDHA and 14-HDHA were not significantly affected by the different beverages and were, respectively, $537 \pm 40$ $\mathrm{pmol} / \mathrm{L}$ and $4641 \pm 697 \mathrm{pmol} / \mathrm{L}$ after water, $528 \pm 44 \mathrm{pmol} / \mathrm{L}$ and $3706 \pm 755 \mathrm{pmol} / \mathrm{L}$ after DRW, and $534 \pm 44 \mathrm{pmol} / \mathrm{L}$ and $3800 \pm 737 \mathrm{pmol} / \mathrm{L}$ after RW. The E-series resolvins, PD1, 10S, 17S-DHDHA, and MaR-1 were not detected.

\section{Relationship between 20-HETE and SPMs}

We examined the relationship between 20-HETE and SPMs because cytochrome P450 enzymes are common to the biosynthesis of both families of compounds. Urinary 20HETE was positively correlated with plasma 18 -HEPE $(\rho=0.32, P=0.004)$. Using a linear mixed model analysis we examined the linear relationship between urinary 20HETE and 18-HEPE whilst consuming different beverages (Supplementary Table 2). After adjusting for 18-HEPE at baseline the slope of the relationship between urinary 20-HETE and 18-HEPE was significantly increased when drinking RW compared with water $(P=0.008)$. The relationship between DRW and water was not different (Supplementary Figure 1). There were no significant relationships between urinary 20HETE and plasma RvD1 or 17-RvD1 that were affected by drinking different beverages.

\section{DISCUSSION}

This study in men has shown for the first time that consuming $375 \mathrm{ml}$ of red wine/day 
for 4 weeks increases blood pressure, vasoconstrictor CYP450 eicosanoids (20-HETE) and oxidative stress ( $\mathrm{F}_{2}$-isoprostanes), while simultaneously increasing SPMs that mediate resolution of inflammation. The effects appear to be due to the alcohol content of the red wine, as effects attributed to consumption of polyphenols in de-alcoholised red wine were not different from those when drinking water. The study demonstrates that moderate-to-high alcohol consumption in men affects eicosanoid synthesis in a complex manner, suggesting that these pathways are important mechanisms relevant to the effects of alcohol on blood pressure and inflammatory responses.

The study showed a modest increase in $24 \mathrm{hr}$ systolic and diastolic BP after 4 weeks of drinking alcohol provided as red wine. The increase was mainly due to an increase in BP while awake. The magnitude of the BP increase after RW was comparable to our previous study where a similar quantity of alcohol was provided as beer or red wine (Zilkens et al., 2005). The $1.4 \mathrm{mmHg}$ increase in $24 \mathrm{hr}$ SBP after drinking red wine may have significant relevance at a population level. The Atherosclerosis Risk in Communities Study (ARIC) suggests that a population-wide strategy to reduce systolic BP by $1 \mathrm{mmHg}$ would result in substantial reductions in heart failure, stroke and coronary heart disease (Hardy et al., 2015). Therefore, public health campaigns to reduce alcohol intake should be promoted to reduce CVD at a population level. There were no independent effects of red wine polyphenols per se (DRW) on systolic or diastolic BP. This finding agrees with our previous studies in normotensive men (Zilkens et al., 2005), premenopausal women (Mori et al., 2015), and patients with Type 2 diabetes (Mori et al., 2016) as well as those in patients with high-normal blood pressure and hypertension (Botden et al., 2012). However, the results are in contrast with the study of Chiva-Blanch and colleagues (2012) who showed a similar quantity of 
polyphenols lowered BP in diabetics or individuals with 3 or more cardiovascular risk factors. This could be due to the fact that our studies (Mori et al., 2015; 2016; Zilkens et al., 2005) and that of Botden et al. (2012) measured 24h ambulatory BP, whereas the study of Chiva-Blanch measured BP three times at 5-min intervals at the end of each intervention.

Our study showed an increase in $24 \mathrm{hr}$ heart rate during RW consumption that was similar to that previously reported by us in normotensive men (Zilkens et al., 2005). There was also a reduction in $24 \mathrm{hr}$ heart rate during consumption of de-alcoholised red wine. The latter finding agrees with a meta-analysis that showed grape seed polyphenols reduced heart rate (Feringa et al., 2011). It is noteworthy that the grape polyphenol intake of the DRW (856 mg/day) in our study was within range of studies included in the meta-analysis (range 150-2000 $\mathrm{mg} /$ day).

There was a significant increase in the vasoconstrictor CYP450 eicosanoid 20-HETE in plasma and urine after drinking red wine. Levels of total plasma EETs, vasodilators formed by CYP450 epoxygenases, and their soluble epoxide hydroxylase metabolites (DHETs) (Figure 1), were unaffected by consumption of red wine. The increase in plasma 20-HETE but not EETs agrees with our previous finding following the acute ingestion of red wine (Barden et al., 2013). 20-HETE has been shown to play a role in inflammation by stimulating the synthesis of pro-inflammatory mediators (Ishizuka et al., 2008).

We observed effects of red wine on oxidative stress with an increase in plasma but not urine $\mathrm{F}_{2}$-isoprostanes, a finding that is consistent with our study in moderate-to-heavy drinkers where alcohol was consumed as beer (Barden et al., 2007). 
The increase in oxidative stress and 20-HETE are potential mechanisms contributing to the increase in blood pressure after alcohol. Future studies examining sodium balance during alcohol consumption are needed to determine if the natriuretic actions of 20HETE in the kidney (Roman, 2002) offset its vasoconstrictor effects thereby attenuating the increase in blood pressure after alcohol.

We did not observe any differential effect of red wine on the inflammatory marker hs$\mathrm{CRP}$, an acute phase reactant produced in the liver in response to inflammation. This agrees with a meta-analysis that pooled the results of five intervention studies and found no significant effect of alcohol on CRP levels (Brien et al., 2011).

This is the first intervention in humans reporting an increase in plasma SPMs without n3 supplementation. We found that red wine increased 18-HEPE that is formed by the actions of CYP450 and/or COX2 on EPA. We also found that RvD1 and 17R-RvD1 that derive from DHA were significantly increased. These findings are significant given that the SPMs increased by red wine are all known to be biologically active (Serhan et al., 2015b). 18-HEPE has been shown to reduce maladaptive cardiac remodelling in mice (Endo et al., 2014). RvD1 and 17R-RvD1 have been implicated in the control of inflammatory pain (Serhan et al., 2015a). In experimental models, RvD1 has been shown to accelerate wound healing in diabetes, prevent colitis, and inhibit kidney fibrosis (Barden et al., 2016), as well as improving ventricular function following myocardial infarction (Kain et al., 2015).

We had hypothesised alcohol could affect synthesis of SPMs by altering the activity of key enzymes involved in SPM synthesis. In regression analysis we found that the slope of the linear association between urinary 20-HETE and plasma 18-HEPE was 
significantly greater when drinking red wine. Alcohol is known to stimulate the CYP450 2E1 enzyme (Pelkonen et al., 1998); therefore, our results could suggest that other CYP450 enzymes involved in the synthesis of both 20-HETE and 18-HEPE are also stimulated by the alcohol in red wine. This is supported by our finding that there was no significant relationship between 20-HETE and RvD1 or 17R-RvD1, as both of these SPM are formed independently of CYP450 enzymes. It is possible that the increase in RvD1 and 17R-RvD1 could be due to an effect of alcohol to stimulate 5lipoxygenase (Guo et al., 2011).

The strengths of this study are that it examined the independent effects of alcohol and polyphenols on BP and a comprehensive range of CYP450 eicosanoids, markers of oxidative stress and inflammation, and mediators of inflammation resolution that may be relevant to the effect of alcohol on CVD risk. The limitations of the study are that the study population was confined to men who were regular drinkers with moderate-to-high alcohol intake. Therefore, our results cannot be extrapolated to males consuming lower amounts of alcohol or to female drinkers.

This study has shown that moderate-to-high alcohol consumption as red wine for 4 weeks increases BP, 20-HETE, and oxidative stress, whilst also increasing a number of SPM associated with the resolution of inflammation. These paradoxical findings require further studies to elucidate whether different CYP450 enzymes are involved, and whether the findings can be replicated in females. The implication of these findings for the effects of alcohol on blood pressure and coronary artery disease warrant further investigation. 


\section{ACKNOWLEDGEMENTS:}

We thank Noelene Atkins and Adeline Indrawan for their assistance with volunteer recruitment and analysis of cytochrome P450 metabolites, respectively.

DISCLOSURES: None of the authors has a conflict of interest to declare. 


\section{References}

AbuAmsha R, Croft KD, Puddey IB, Proudfoot JM, Beilin LJ (1996) Phenolic content of various beverages determines the extent of inhibition of human serum and low-density lipoprotein oxidation in vitro: Identification and mechanism of action of some cinnamic acid derivatives from red wine. Clin Sci 91:449-458.

Barden A, Mas E, Croft KD, Phillips M, Mori TA (2014) Short-term n-3 fatty acid supplementation but not aspirin increases plasma proresolving mediators of inflammation. J Lipid Res 55:2401-2407.

Barden A, Zilkens RR, Croft K, Mori T, Burke V, Beilin LJ, Puddey IB (2007) A reduction in alcohol consumption is associated with reduced plasma F2isoprostanes and urinary 20-HETE excretion in men. Free Radic Biol Med 42:1730-1735.

Barden AE, Croft KD, Beilin LJ, Phillips M, Ledowski T, Puddey IB (2013) Acute effects of redwine on cytochrome P450 eicosanoids and blood pressure in men. $\mathrm{J}$ Hypertens 31:2195-2202.

Barden AE, Mas E, Mori TA (2016) n-3 Fatty acid supplementation and proresolving mediators of inflammation. Curr Opin Lipidol 27:26-32.

Botden IPG, Draijer R, Westerhof BE, Rutten JHW, Langendonk JG, Sijbrands EJG, Danser AHJ, Zock PL, van den Meiracker AH (2012) Red wine polyphenols do not lower peripheral or central blood pressure in high normal blood pressure and hypertension. Am J Hypertens 25:718-723.

Brien SE, Ronksley PE, Turner BJ, Mukamal KJ, Ghali WA (2011) Effect of alcohol consumption on biological markers associated with risk of coronary heart 
disease: systematic review and meta-analysis of interventional studies. Brit Med J 342.

Caccetta RAA, Croft KD, Beilin LJ, Puddey IB (2000) Ingestion of red wine significantly increases plasma phenolic acid concentrations but does not acutely affect ex vivo lipoprotein oxidizability. Am J Clin Nutr 71:67-74.

Chiva-Blanch G, Urpi-Sarda M, Ros E, Arranz S, Valderas-Martinez P, Casas R, Sacanella E, Llorach R, Lamuela-Raventos RM, Andres-Lacueva C, Estruch R (2012) Dealcoholized red wine decreases systolic and diastolic blood pressure and increases plasma nitric oxide. Circ Res 111:1065-+.

Endo J, Sano M, Isobe Y, Fukuda K, Kang JX, Arai H, Arita M (2014) 18-HEPE, an n3 fatty acid metabolite released by macrophages, prevents pressure overloadinduced maladaptive cardiac remodeling. J Exp Med 211:1673-1687.

Feringa HHH, Laskey DA, Dickson JE, Coleman CI (2011) The Effect of Grape Seed Extract on Cardiovascular Risk Markers: A meta-analysis of randomized controlled trials. J Am Diet Assoc 111:1173-1181.

Friedewald.WT, Fredrick.DS, Levy RI (1972) Estimation of concentration of lowdensity lipoprotein cholesterol in plasma, without use of preparative ultracentrifuge. Clin Chem 18:499-\&.

Guo Y, Wang X, Zhang X, Sun Z, Chen X (2011) Ethanol promotes chemically induced oral cancer in mice through activation of the 5-lipoxygenase pathway of arachidonic acid metabolism. Cancer Prev Res 4:1863-1872.

Hardy ST, Loehr LR, Butler KR, Chakladar S, Chang PP, Folsom AR, Heiss G, MacLehose RF, Matsushita K, Avery CL (2015) Reducing the blood pressure- 
related burden of cardiovascular disease: Impact of achievable improvements in blood pressure prevention and control. JAMA 4.

Hoff U, Lukitsch I, Chaykovska L, Ladwig M, Arnold C, Manthati VL, Fuller TF, Schneider W, Gollasch M, Muller DN, Flemming B, Seeliger E, Luft FC, Falck JR, Dragun D, Schunck WH (2011) Inhibition of 20-HETE synthesis and action protects the kidney from ischemia/reperfusion injury. Kidney Int 79:57-65.

Hoopes SL, Garcia V, Edin ML, Schwartzman ML, Zeldin DC (2015) Vascular actions of 20-HETE. Prostag Oth Lipid M 120:9-16.

Imhof A, Froehlich M, Brenner H, Boeing H, Pepys MB, Koenig W (2001) Effect of alcohol consumption on systemic markers of inflammation. Lancet 357:763767.

Ishizuka T, Cheng J, Singh H, Vitto MD, Manthati VL, Falck JR, LaniadoSchwartzman M (2008) 20-hydroxyeicosatetraenoic acid stimulates nuclear factor-kappa B activation and the production of inflammatory cytokines in human endothelial cells. J Pharmacol ExpTher 324:103-110.

Kain V, Ingle KA, Colas RA, Dalli J, Prabhu SD, Serhan CN, Joshi M, Halade GV (2015) Resolvin D1 activates the inflammation resolving response at splenic and ventricular site following myocardial infarction leading to improved ventricular function. J Mol Cell Cardiol 84:24-35.

Kim SY, Breslow RA, Ahn J, Salem N, Jr. (2007) Alcohol consumption and fatty acid intakes in the 2001-2002 national health and nutrition examination survey. Alcohol Clin Exp Res 31:1407-1414.

Klatsky AL (2015) Alcohol and cardiovascular diseases: where do we stand today? J Intern Med 278:238-250. 
Knapp DJ, Crews FT (1999) Induction of cyclooxygenase-2 in brain during acute and chronic ethanol treatment and ethanol withdrawal. Alcohol Clin Exp Res 23:633-643.

Mas E, Croft KD, Zahra P, Barden A, Mori TA (2012) Resolvins D1, D2, and other mediators of self-limited resolution of inflammation in human blood following n-3 fatty acid supplementation. Clin Chem 58:1476-1484.

Mori TA, Burke V, Beilin LJ, Puddey IB (2015) Randomized Controlled Intervention of the Effects of Alcohol on Blood Pressure in Premenopausal Women. Hypertension 66:517-523.

Mori TA, Burke V, Zilkens RR, Hodgson JM, Beilin LJ, Puddey IB (2016) The effects of alcohol on ambulatory blood pressure and other cardiovascular risk factors in type 2 diabetes: a randomized intervention. J Hypertens 34:421-428.

Pelkonen O, Maenpaa J, Taavitsainen P, Rautio A, Raunio H (1998) Inhibition and induction of human cytochrome P450 (CYP) enzymes. Xenobiotica 28:12031253.

Roman RJ (2002) P-450 metabolites of arachidonic acid in the control of cardiovascular function. Physiol Rev 82:131-185.

Salmela KS, Kessova IG, Tsyrlov IB, Lieber CS (1998) Respective roles of human cytochrome P-4502E1, 1A2, and 3A4 in the hepatic microsomal ethanol oxidizing system. Alcohol Clin Exp Res 22:2125-2132.

Serhan CN, Chiang N, Dalli J (2015a) The resolution code of acute inflammation: Novel pro-resolving lipid mediators in resolution. Sem Immunol 27:200-215.

Serhan CN, Chiang N, Dalli J, Levy BD (2015b) Lipid Mediators in the Resolution of Inflammation. Cold Spring Harbor Perspectives in Biology 7. 
Toth P, Csiszar A, Sosnowska D, Tucsek Z, Cseplo P, Springo Z, Tarantini S, Sonntag WE, Ungvari Z, Koller A (2013) Treatment with the cytochrome P450hydroxylase inhibitor HET0016 attenuates cerebrovascular inflammation, oxidative stress and improves vasomotor function in spontaneously hypertensive rats. Brit J Pharmacol 168:1878-1888.

Tsai IJ, Croft KD, Mori TA, Falck JR, Beilin LJ, Puddey IB, Barden AE (2009) 20HETE and F2-isoprostanes in the metabolic syndrome: the effect of weight reduction. Free Radic Biol Med 46:263-270.

Ward NC, Puddey IB, Hodgson JM, Beilin LJ, Croft KD (2005) Urinary 20hydroxyeicosatetraenoic acid excretion is associated with oxidative stress in hypertensive subjects. Free Radic Biol Med 38:1032-1036.

Wheelan P, Murphy RC (1997) Quantitation of 5-lipoxygenase products by electrospray mass spectrometry: Effect of ethanol on zymosan-stimulated production of 5lipoxygenase products by human neutrophils. Analyt Biochem 244:110-115.

Zilkens RR, Burke V, Hodgson JM, Barden A, Beilin LJ, Puddey IB (2005) Red wine and beer elevate blood pressure in normotensive men. Hypertension 45:874-879. 


\section{Figure Legends}

Figure 1. A schematic overview of bioactive eicosanoids from arachidonic acid (AA), eicosapentaenoic acid (EPA) and docosahexaenoic acid (DHA) involved in vascular function and inflammation resolution. AA is metabolised by:- cyclooxygenase (COX-1 and 2) forming prostaglandins (PG's) and thromboxane $\mathrm{A}_{2}\left(\mathrm{TXA}_{2}\right)$; lipoxygenases (5$\mathrm{LOX}$ ) to leukotriene $\mathrm{B}_{4}\left(\mathrm{LTB}_{4}\right)$, and (5-LOX and 15-LOX) to lipoxins ( $\left.\mathrm{LXA}_{4} \& \mathrm{LXB}_{4}\right)$; cytochrome (CYP) P450s to 20-hydroxyeicosatetraenoic acid (20-HETE) and epoxyeicosatrienoic acids (EETs). EETs are inactivated by soluble epoxide hydrolase (sEH) forming dihydroxyeicosatrienoic acids (DHETs). AA also undergoes nonenzymatic free radical oxidation to $\mathrm{F}_{2}$-isoprostanes. EPA is metabolised by COX2/CYP450 to 18-HEPE with further metabolism by 5-LOX and 15-LOX forming Eseries resolvins (RvE1-RvE3). DHA metabolism by COX-2/15-LOX results in formation 17-HDHA that is further metabolised to PD1, 10S,17S-DHDHA and D-series resolvins. DHA metabolism by 12-LOX leads to the Maresins (MaR-1 and 14-HDHA). The eicosanoids in black boxes denote those measured in this study.

Figure 2. Ambulatory systolic blood pressure (SBP) (a) diastolic blood pressure (DBP) (b) and heart rate (c) over $24 \mathrm{~h}$ after 4 weeks consuming water $(\square)$, DRW ( $\Delta$ ) or RW (•). RW significantly increased SBP $(P=0.003)$, DBP $(P=0.032)$ and heart rate $(P=0.0001)$ over the $24 \mathrm{~h}$ compared with DRW or water. Heart rate was lower after $\operatorname{DRW}(P=0.001)$ compared with water.

Figure 3. CYP 450 eicosanoids: urinary 20-HETE (a), plasma 20-HETE (b), plasma EETs (c) and plasma DHETs (d) and markers of oxidative stress plasma $\mathrm{F}_{2}$-isoprostanes (e) and urinary $\mathrm{F}_{2}$-isoprostanes (f) after consuming water (white bars), DRW (grey bars) 
or RW (black bars). Urinary and plasma 20-HETE were higher after RW compared with DRW, $† P<0.01,{ }^{*} P<0.05$. Plasma $\mathrm{F}_{2}$-isoprostanes was higher after $\mathrm{RW}$ compared with DRW and water $+P<0.001$

Figure 4. Plasma SPM: 18-HEPE (a) RvD1 (b) and 17R-RvD1 (c) after consuming water (white bars), DRW (grey bars) or RW (black bars). Plasma SPM were significantly higher after RW compared with DRW and water. $* P<0.05$. 
Table 1.

\begin{tabular}{lc}
\hline Characteristic & Mean \pm SD \\
\hline Age (yrs) & $54.1 \pm 6.6$ \\
Average Alcohol intake (g/day) & $52.6 \pm 20.5$ \\
BMI (Kg/m ${ }^{2}$ ) & $27.5 \pm 2.9$ \\
SBP (mmHg) & $127 \pm 9$ \\
DBP (mmHg) & $77 \pm 5$ \\
Cholesterol (mmol/L) & $5.3 \pm 0.9$ \\
Triglycerides (mmol/L) & $1.1 \pm 0.7$ \\
LDL-cholesterol (mmol/L) & $3.3 \pm 0.7$ \\
HDL-cholesterol (mmol/L) & $1.5 \pm 0.5$ \\
Glucose (mmol/L) & $4.8 \pm 0.5$ \\
Creatinine ( $\mu$ mol/L) & $88 \pm 13$ \\
$\gamma$-glutamyl transpeptidase (U/L) & $33 \pm 21$ \\
Alanine transaminase (U/L) & $32 \pm 13$ \\
Aspartate transaminase (U/L) & $29 \pm 5$ \\
\hline
\end{tabular}

Blood pressure measured at screening using Dinamap Pro 200. Values are mean \pm SD. 
Table 2.

\begin{tabular}{|c|c|c|c|c|}
\hline & Baseline & Water & DRW & RW \\
\hline \multicolumn{5}{|c|}{ Systolic BP (mmHg) } \\
\hline \multirow[t]{2}{*}{$24 \mathrm{~h}$} & 127.6 & 128.1 & 127.7 & $129.1^{\mathrm{a}}$ \\
\hline & $(124.4,130.9)$ & $(124.9,131.3)$ & $(124.4,130.9)$ & $(125.9,132.3)$ \\
\hline \multirow[t]{2}{*}{ Awake } & 132.3 & 133.7 & 133.0 & $134.7^{\mathrm{a}}$ \\
\hline & $(128.9,135.8)$ & $(130.3,137.2)$ & $(129.6,136.5)$ & $(131.2,138.1)$ \\
\hline \multirow[t]{2}{*}{ Asleep } & 116.3 & 114.9 & 114.7 & 116.0 \\
\hline & $(113.1,119.5)$ & $(111.7,118.1)$ & $(111.9,118.4)$ & $(112.8,119.2)$ \\
\hline \multicolumn{5}{|c|}{ Diastolic BP (mmHg) } \\
\hline \multirow[t]{2}{*}{$24 \mathrm{~h}$} & 79.2 & 79.5 & 79.9 & $80.7^{\mathrm{b}}$ \\
\hline & $(76.6,81.8)$ & $(76.9,82.0)$ & $(77.3,82.4)$ & $(78.1,83.3)$ \\
\hline \multirow[t]{2}{*}{ Awake } & 83.2 & 84.2 & 84.5 & $85.5^{b}$ \\
\hline & $(80.4,85.9)$ & $(81.4,86.9)$ & $(81.8,87.2)$ & $(82.8,88.2)$ \\
\hline \multirow[t]{2}{*}{ Asleep } & 69.4 & 68.1 & 68.9 & 69.2 \\
\hline & $(66.7,72.2)$ & $(65.4,71.9)$ & $(66.2,71.7)$ & $(66.5,71.9)$ \\
\hline \multicolumn{5}{|c|}{ Heart Rate (beats/min) } \\
\hline \multirow[t]{2}{*}{$24 \mathrm{~h}$} & 63.0 & 65.7 & $63.7^{c}$ & $68.4^{\mathrm{a}}$ \\
\hline & $(59.3,66.7)$ & $(62.0,69.4)$ & $(59.9,67.4)$ & $(64.7,72.1)$ \\
\hline \multirow[t]{2}{*}{ Awake } & 66.0 & 68.9 & $66.8^{c}$ & $70.6^{\mathrm{a}}$ \\
\hline & $(62.1,70.0)$ & $(64.9,72.9)$ & $(62.8,70.7)$ & $(66.6,74.6)$ \\
\hline \multirow[t]{2}{*}{ Asleep } & 56.1 & 58.2 & $56.4^{c}$ & $63.3^{\mathrm{a}}$ \\
\hline & $(52.6,59.6)$ & $(54.7,61.9)$ & $(52.9,59.9)$ & $(59.9,66.8)$ \\
\hline
\end{tabular}

Values are means and 95\% confidence intervals. ${ }^{\mathrm{a}} P<0.01,{ }^{\mathrm{b}} P<0.05$ compared with water and DRW, ${ }^{\mathrm{c}} P<0.01$ compared with water. 


\section{Table Legends}

Table 1. Baseline characteristics of the 22 men studied

Table 2. Mean 24h, awake and asleep systolic BP, diastolic BP and heart rate at baseline and after 4 weeks of consuming either water, DRW or RW 
Figure 1

Figure 2
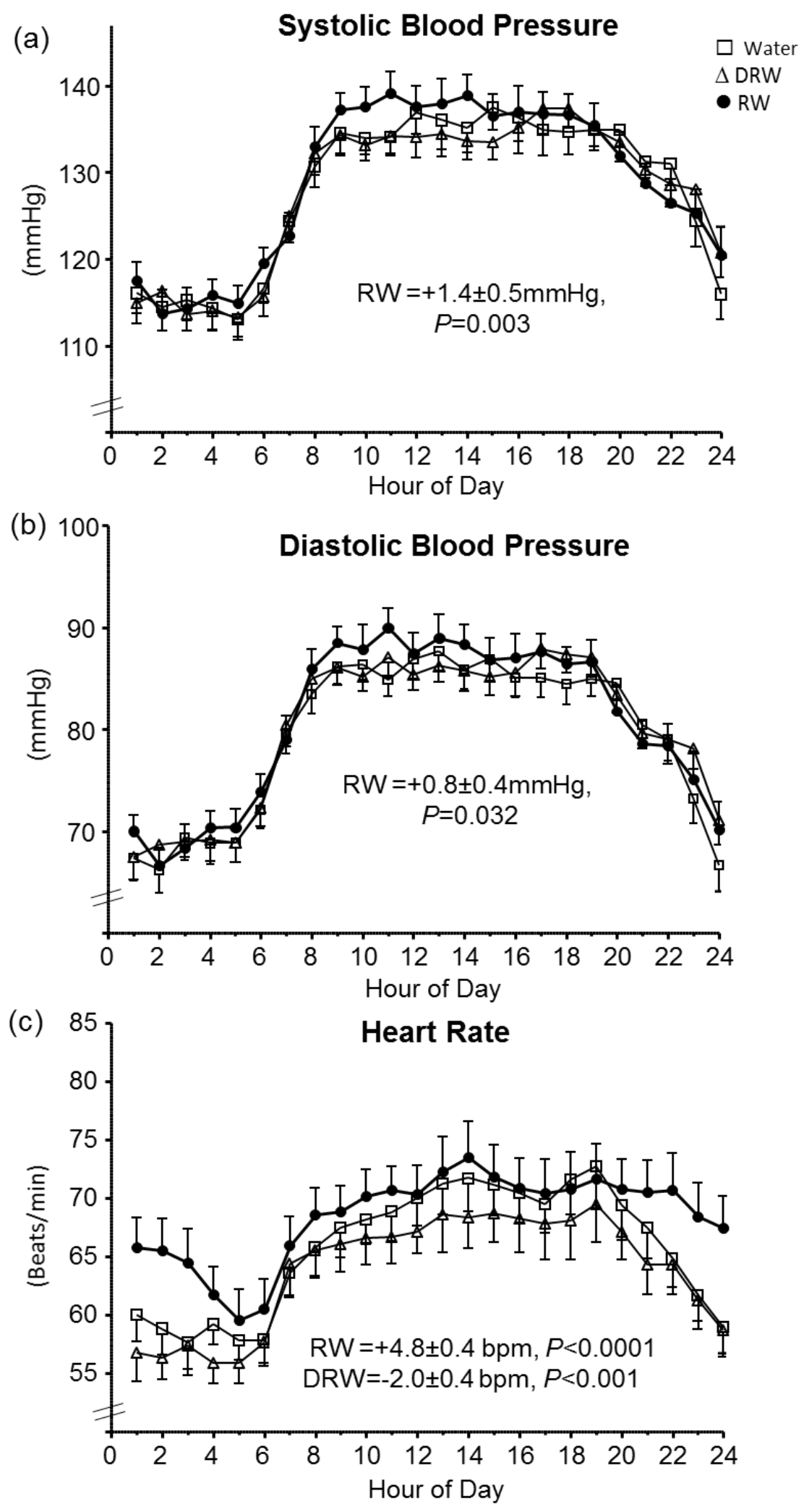
Figure 3
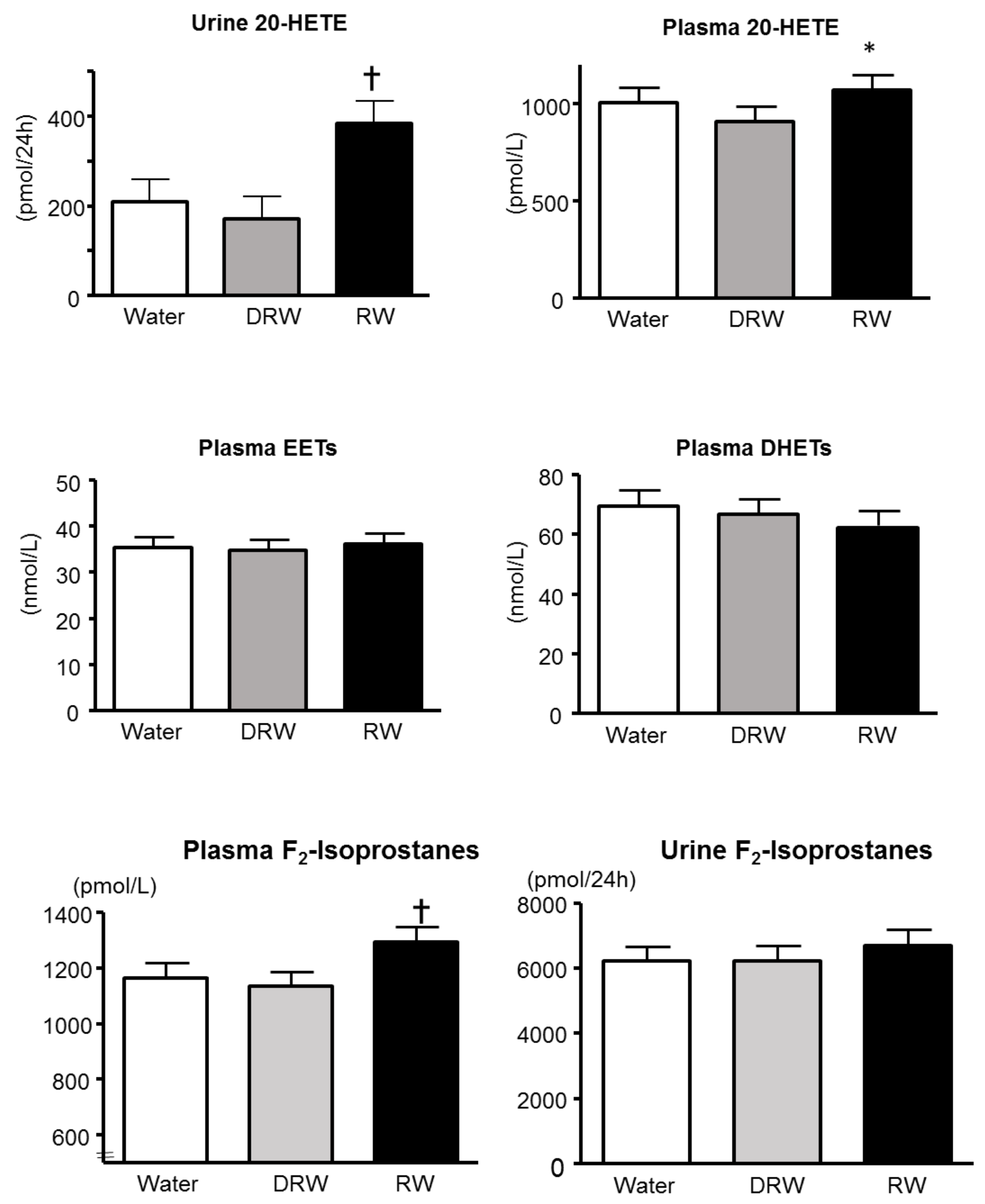
Figure 4

\section{Plasma SPM}
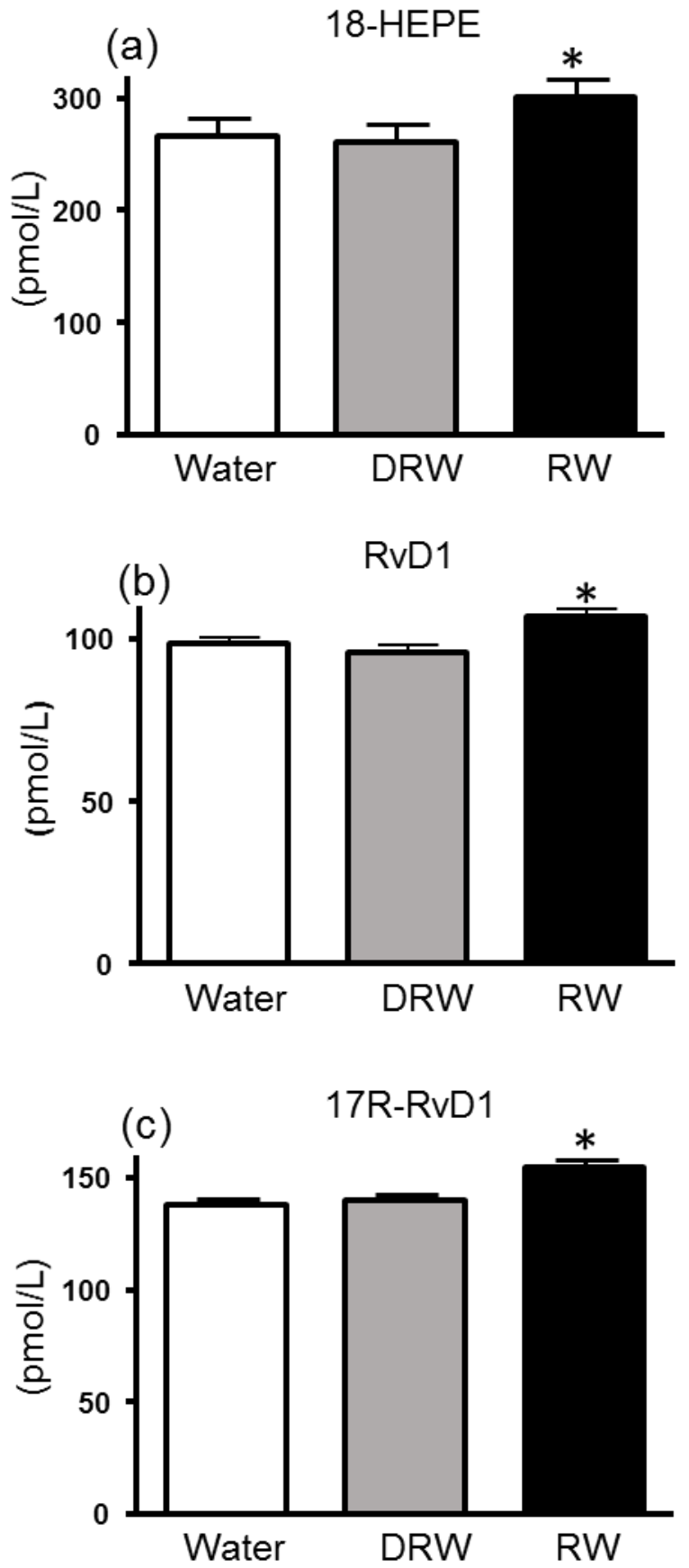